\title{
Ovarian Cancer Revealed by Paraneoplastic Cerebellar Degeneration Anti-Yo Positive: A Case Report
}

\author{
Hayat Erraichi ${ }^{\text {"1 }}$, Niaina Ezra Randriamanovontsoa ${ }^{1}$, Valère Litique ${ }^{1}$, Jean-Marc Limacher ${ }^{1}$, \\ S. Berrad ${ }^{2}$, Karima Oualla ${ }^{2}$, Lamiae Ammadour ${ }^{2}$, Zineb Benbrahim ${ }^{2}$, S. Arifi ${ }^{2}$, Nawfal Mellas ${ }^{2}$ \\ ${ }^{1}$ Onco-Hematology Service, Louis Pasteur Hospital Center,39 avenue de la Liberté, 68000 Colmar, France. \\ ${ }^{2}$ Medical Oncology Service, CHU Hassan II fes, Morocco
}

Corresponding author: Hayat Erraichi; erraichierraichi@gmail.com

Received: 02 December 2020;

Accepted: 15 December 2020;

Published: 20 December 2020

\section{Summary}

Neurological paraneoplastic syndromes are rare, often associated with gynecological cancer or small cell lung cancer. This article reports a case of ovarian cancer to discuss the difficulties in the management of neurological paraneoplastic syndromes.

This is a case of paraneoplastic cerebellar syndrome with anti-Yo antibodies. Neurological syndromes, testing for onconeural antibodies and testing for underlying cancer provide a basis for the diagnosis. The anti-tumor treatment constitutes the mainstay of the care.

Keywords: neurological paraneoplastic syndromes, ovarian cancer, onconeural antibodies.

\section{Introduction}

Paraneoplastic neurological syndrome (PNS) is defined by the occurrence of a neurological syndrome which cannot be explained by a metastatic, iatrogenic, toxic or deficient aetiology. There are several clinical presentations of which the most reported in the literature are: paraneoplastic cerebellar degeneration (PCD), limbic encephalitis, encephalomyelitis, subacute sensory neuropathy, Lambert Eaton's syndrome, opsoclonus-myoclonus, dermatopolymyositis and pseudo-occlusive syndrome. (DCP) complicates many tumors such as breast cancer, lung cancer and some gynecological cancers. We report here a case of ovarian cancer discovered following PNS in order to discuss the diagnostic difficulty and therapeutic.

\section{Case présentation}

55-year-old patient with a history of arterial hypertension, admitted to the Neurology Department for rapidly progressive worsening static and kinetic cerebellar syndrome. On admission, the patient was conscious, well oriented, responding appropriately but slowly to a random question. The patient presented also a cerebellar dysarthria, major dysmetria predominantly in the upper limbs, with volitional dyskinesias, adiadokokinesia, asynergy, hypotonia of the lower limbs and abduction attitude with babinski on the right, bilateral vertical and rotatory nystagmus and in both lateral gazes, ocular fixation impossible. Cerebral MRI performed did not show any abnormalities in the intra-axial structures of the posterior cerebral fossa, Electroneuromyogram was in favor of a sensitive neuronopathy of the 4 limbs. The search for anti-neuronal antibodies: anti-Hu, anti-Ri, anti -GAD, anti-GABA-B, antiNMDAR, anti-AMPAR, ANTI -gangliosides, anti-sulfatides in blood and cephalospinal fluid was negative. The anti-Yo antibodies, on the other hand, were positive. The Thoraco abdomino pelvic scanner revealed a heterogeneous bilateral ovarian tumor $(65 \times 42 \mathrm{~mm}$ on the right $-26 \times 10 \mathrm{~mm}$ on the left) with left external iliac lymphadenopathy, bilateral primary iliac and retroperitoneal lymphadenopathy (figure 1).

PET scan showed 2 intensely hyper metabolic bilateral ovarian masses right $44 \mathrm{~mm}$ (SUV $\max =40.2$ ); left (SUV $\max =$ 16).

The CA125 was $245 \mathrm{IU} / \mathrm{ml}$, the patient underwent a total hysterectomy + adnexectomy + dissection + omentectomy + peritoneal cytology, the pathology anatomy concluded in a grade III endometrioid adenocarcinoma of tubo-ovarian origin.

The hypothesis of a paraneoplastic cerebellar degeneration was retained. Adjuvant chemotherapy with carboplatin and paclitaxel was administered and the thoraco-abdomino-pelvic scan showed no signs of progressive recovery.

On the neurological level, the patient benefited from 2 curures despite the treatment with polyvalent immunoglobulins and corticosteroids without clinical improvement. The patient is still severely hampered by a cerebellar syndrome whose recovery seems to be stagnating. 


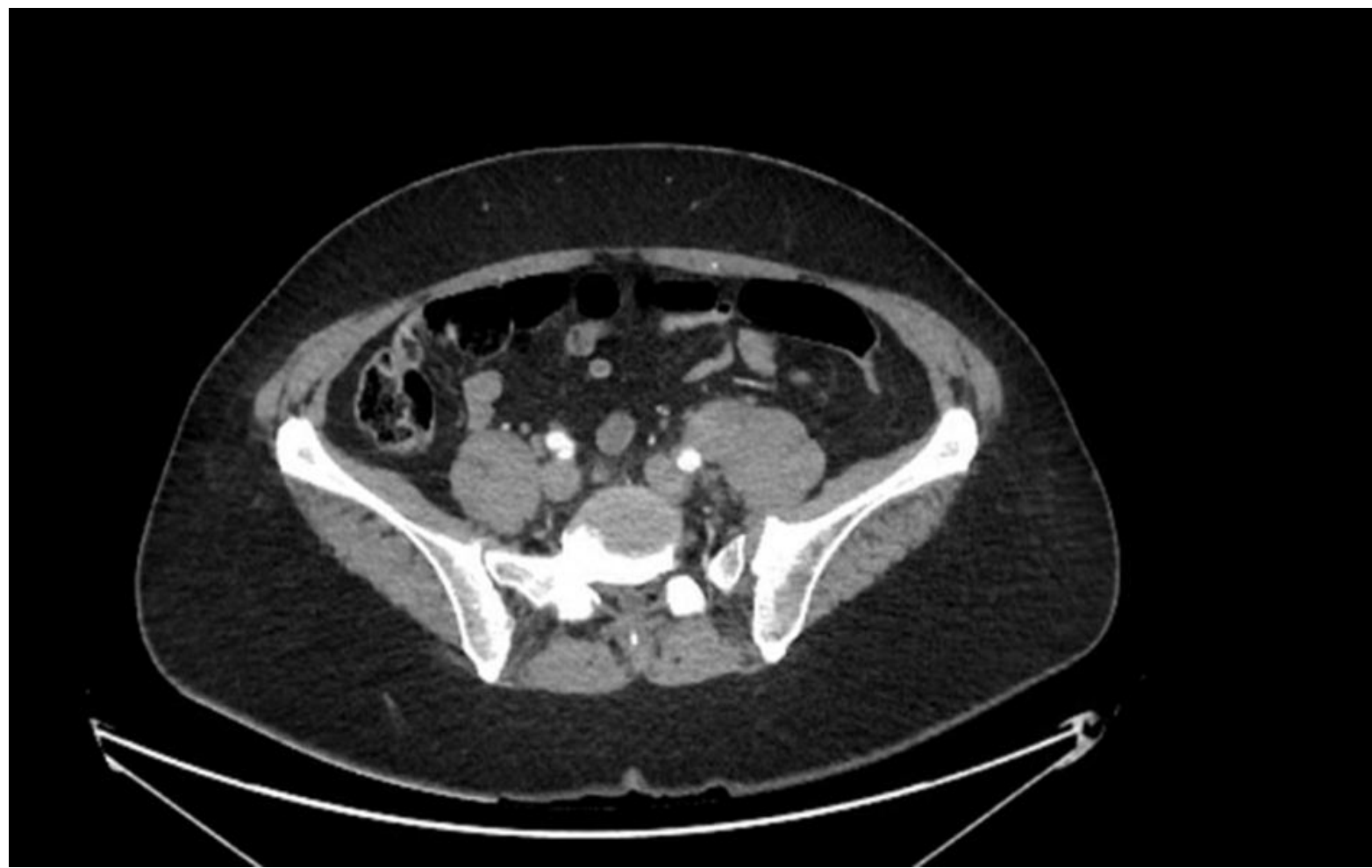

Figure 1: Pelvic CT scan showing a heterogeneous bilateral ovarian tumor $(65 \times 42 \mathrm{~mm}$ on the right $-26 \times 10 \mathrm{~mm}$ on the left) with left external iliac, bilateral primary iliac and retro-peritoneal lymphadenopathy

\section{Discussion}

This reported case illustrates a frequently encountered SPN is cerebellar degeneration. The triad of clinical neuralgic syndromes, specific autoantibody positivity and tumor background was complete. Our case had cerebellar syndrome; anti-Yo antibodies were positive in serum and CSF.

The clinical presentations of SNP can be diverse but eight classic syndromes should attract the attention of clinicians: limbic encephalitis, acute or subacute cerebellar degeneration, encephalomyelitis, opsoclonus myoclonus, acute sensory neuropathy, de Lambert-Eaton, pseudo-occlusive syndrome and dermatopolymyositis. The manifestations are most often subacute or acute, in a tumor context and in a patient with major risk factors for cancer ${ }^{[1]}$.

PCD is most often associated with lung and gynecological cancers, the incidence and prevalence of which remain unknown. It affects both women and men. The mean age of onset of PCD reflects the age of onset of cancers ${ }^{[2]}$. Symptoms are associated with bilateral static and kinetic cerebellar syndrome and dysarthria. Dizziness and nystagmus can be observed ${ }^{[3]}$. The pathophysiology of PCD is poorly studied. The hypothesis of an autoimmune mechanism involved has been suggested. It is believed to be a cross reaction due to ectopic expression by the tumor of proteins normally expressed by the nervous system. The presence of circulating autoantibodies (serum and cerebrospinal fluid (CSF)), specifically associated with PNS, is one of the characteristics of these syndromes. There are two types of SNP depending on the target of the antibodies associated with them and which can be directed against intracellular (onconeuronal) or membrane targets. Among the onconeuronal antibodies identified in DCP we find: Hu, Yo, CV2, Ri, Ma, Tr / DNER, amphiphysine, Sox ${ }^{\left[{ }^{[4]}\right.}$. Anti-Yo antibodies are associated with certain gynecological (ovarian and rarely uterus), breast, lung and gastric cancers. The presence of anti-Yo antibodies in a woman presenting with a cerebellar syndrome is in $90 \%$ of cases associated with breast or ovarian cancer. The discovery of these antibodies should encourage research into this type of tumor ${ }^{[5]}$. Treatment of PCD is based on the management of the primary tumor. The difficulty of care also lies in the therapeutic aspect. Probably due to the rarity of SPN, no treatment protocol has been established so far. However, in the reported cases two therapeutic approaches emerge: treatment of the underlying cancer if it is diagnosed and suppression of the immune response ${ }^{[6]}$. Antitumor treatment is the mainstay of treatment, which stabilizes or eliminates neurological signs and improves the prognosis. Symptomatic treatment without direct action on the tumor seems uncertain ${ }^{[6,7,8,9]}$. The combination of anti-tumor treatment with immunotherapy would give good results, especially in the acute phase of the symptoms. Corticosteroids, cyclophosphamide, tacrolimus, immunoglobulin, plasmapheresis and rituximab are commonly used concomitant treatments. They can be administered as monotherapy or in combination ${ }^{[10]}$.

\section{Conclusion}

PNS should suggest a classic subacute neurological syndrome in a patient with risk factors for cancer. The diagnostic certainty of the underlying tumor of a PNS is often achieved late. Antitumor drugs are the main treatment. The prognosis depends on the rapidity of diagnosis and treatment. In a patient presenting a cerebellar syndrome associated with anti-Yo positive CAs, active screening for gynecological cancer should be performed.

\section{Consent for Publication}

Consent from patient was obtained before publication of this case report.

\section{Availability of Data and Materials}

The data are available from the corresponding author on reasonable request. 


\section{Conflict of Interest Statement}

There are no conflicts of interest to declare.

\section{Bibliographies}

[1] Didelot A, Honnorat J. Les syndromes neurologiques paranéoplasiques. Rev Med Interne 2011; 32: 605-11.

[2] Giometto B, Grisold W, Vitaliani R, Graus F, Honnorat $\mathrm{J}$, Bertolini Get al. Paraneoplastic neurologic syndrome in the PNS Euronetwork data-base: a European study from 20 centers. Arch Neurol. 1998;67(3):330-5.

[3] Gatti G, Simsek S, Kurne A, Zurrida S, Naninato P, Veronesi $\mathrm{P}$ et al. Paraneoplastic neurological disorders in breast cancer. Breast. 2003;12(3):203-7.

[4] Honnorat J, Cartalat-Carel S, Ricard D, Camdessanche JP, Carpentier AF, Rogemond V et al. Onconeural antibodies and tumour type determine survival and neurological symptoms in paraneoplastic neurological syndromes with $\mathrm{Hu}$ or CV2/CRMP5 anti-bodies. J Neurol Neurosurg Psychiatry. 1998;80(4):412-6.

[5] Shams'ili S, Grefkens J, de Leeuw B, van den Bent M, Hooijkaas H, van der Holt B et al. Paraneoplastic cerebellar degeneration associated with antineuronal antibodies: analysis of 50 patients. Brain. $126(\mathrm{Pt}$ 6):1409-18.
[6] Darnell RB, Posner JB. Paraneoplastic syndromes involving the nervous system. N Engl J Med 2003; 349: 1543-54.

[7] Honnorat J. Pathologie dysimmunitaire de la jonction neuro-muscu- laire : le syndrome de Lambert-Eaton. Bull Acad Natle Méd 2014; 192: 243-55.

[8] Dalmau J, Gonzalez RG, Lerwill MF. A 56-Year-Old Woman with Rapidly Progressive Vertigo and Ataxia. N Engl J Med 2007; 356: 612-20.

[9] Titulaer MJ, Wirtz PW, Willems LN, van Kralingen KW, Smitt PA, Verschuuren JJ. Screening for small-cell lung cancer: a follow-up study of patients with Lambert-Eaton myasthenic syndrome. J Clin Oncol 2008; 26: 4276-81.

[10] Keime-Guibert F, Graus F, Fleury A, René R, Honnorat J, Broetv P, Delattre JY. Treatment of paraneoplastic neurological syndromes with antineuronal antibodies (Anti-Hu, Anti-Yo) with a combination of immunoglobulins, cyclophosphamide, and Methylprednisolone. J Neurol Neurosurg Psychiatry 2000; 68: 479-82.

[11] Viaccoz A, Honnorat J. Évolutions conceptuelles des syndromes neurologiques paranéoplasiques. Pratique Neurologique-FMC. 2012;3(1):14-25.

[12] Noorani A, Sadiq Z, Minakaran N, Coleman C, Thomas VA, Mokbel K. Paraneoplastic cerebellar degeneration as a presentation of breast cancer-a case report and review of the literature. Int Semin Surg Oncol. 2008;5:8. 